\title{
Voltammetric biosensors for the determination of paracetamol at carbon nanotube modified pyrolytic graphite electrode
}

\author{
R. N. Goyal ${ }^{a}$, Vinod Kumar Gupta ${ }^{a, b,{ }^{*}, S . \text { Chatterjee }^{a}}$ \\ a Department of Chemistry, Indian Institute of Technology Roorkee, Roorkee 247667, India \\ ${ }^{b}$ KFUPM Chair Professor, Chemistry Department, King Fahd University of Petroleum and Minerals, \\ Dhahran, Saudi Arabia
}

\begin{abstract}
In this paper, we describe the research and development of a method of analysis for the determination of paracetamol by square wave voltammetry (SWV) at carbon nanotubes modified EPPGE. A comparison of the voltammetric signals of paracetamol on SWNT modified EPPGE with MWNT modified EPPGE and bare EPPGE has been made. The procedure has been applied satisfactorily to the determination of paracetamol in different pharmaceutical products and biological fluids. The method is simple, rapid and sensitive and no preparation procedures were required for the analysis of paracetamol. To the best of our knowledge there is no report on using single-walled carbon nanotubes modified edge plane pyrolytic graphite electrodes for determination of paracetamol.
\end{abstract}

\section{Chemical and Biochemical Sensors}

Paracetamol (I) or acetaminophen is an analgesic medicament similar to acetylsalicylic acid lacking anticoagulatory properties and gastric irritation. It is widely used as an active ingredient in pharmaceutical preparations as it is not considered to be carcinogenic at therapeutic doses. Therefore, development of a simple, inexpensive, sensitive and accurate analytical method for determination of paracetamol is of paramount importance. A range of methods have been utilized for determination of paracetamol, alone and in mixtures, in formulations and biological samples, such as high performance liquid chromatography [1] spectrophotometry [2], capillary electrophoresis [3], liquid chromatography-mass spectrometry [4], spectrofluorimetry [5], thin layer chromatography [6] and micellar electrokinetic chromatography [7]. However, these methods suffer from some disadvantages such as long analysis time, high cost, and requirement for sample pretreatment and in some cases low sensitivity and selectivity that makes them unsuitable for routine analysis. Since, paracetamol can be electrochemically oxidized, its determination using electrochemical sensors (Bio Sensors) and methods has received considerable interest in the past few decades [8-11]. Carbon nanotubes modified electrodes have attracted attention in last few years and many biomolecules have been determined at such electrodes. However, to the best of our knowledge there is no report on using singlewalled carbon nanotubes modified edge plane pyrolytic graphite electrodes for determination of paracetamol.

\section{Results}

The voltammetric oxidation of paracetamol on single-walled carbon nanotubes (SWNT) modified edge plane pyrolytic graphite electrode (EPPGE) was explored in phosphate buffer solution by using square wave voltammetry. Cyclic and square wave voltammetry studies indicated the oxidation of paracetamol at the electrode surface through a two-electron reversible step and fundamentally controlled by adsorption. Besides semi-infinite planar diffusion, the role of thin layer diffusion at nanotube modified electrodes is also suggested. A comparison of the voltammetric response of paracetamol at SWNT modified EPPGE and MWNT modified EPPGE (Fig 1) clearly revealed that SWNT acts as a better surface modifier in comparison to MWNT at fixed concentration of nanotubes in $\mathrm{mg} / \mathrm{mL}$ of the solvent. Such a deposition imparts different effective surface areas to electrodes. The significant increase in current response for the ferri/ferro cyanide oxidation at SWNT modified EPPGE cannot account for by the semi-infinite diffusion model alone and can also be attributed to the thin layer diffusion as reported earlier at SWNT modified glassy carbon electrode by Streeter et al. [12].

The improvement of paracetamol voltammetric signal at the modified electrode also suggests the diffusion within porous layer in addition to semi-infinite diffusion. The uncertainty in the 
thin layers formed during casting of nanotube solution, their area and uniform activity of the nanotubes are few important parameters that may affect the diffusion within porous layer.The sensitivity at SWNT modified EPPGE is 2 times more than that at MWNT modified EPPGE. Paracetamol gave a sensitive oxidation peak at $187 \mathrm{mV}$ at $\mathrm{pH} 7.2(\mu=0.5 \mathrm{M})$ which was used to quantitate the drug in the range of $5-1000$ $\mathrm{nM}$ with a detection limit of $2.9 \times 10-9 \mathrm{M}$ at
SWNT modified EPPGE. The interfering effect of physiologically common interferents on the current response of paracetamol has been reported. The procedure was successfully applied for the assay of paracetamol in pharmaceutical formulations. The applicability of the developed method to determine the drug in human urine samples obtained after $4 \mathrm{~h}$ of administration of paracetamol is illustrated (Table 1).

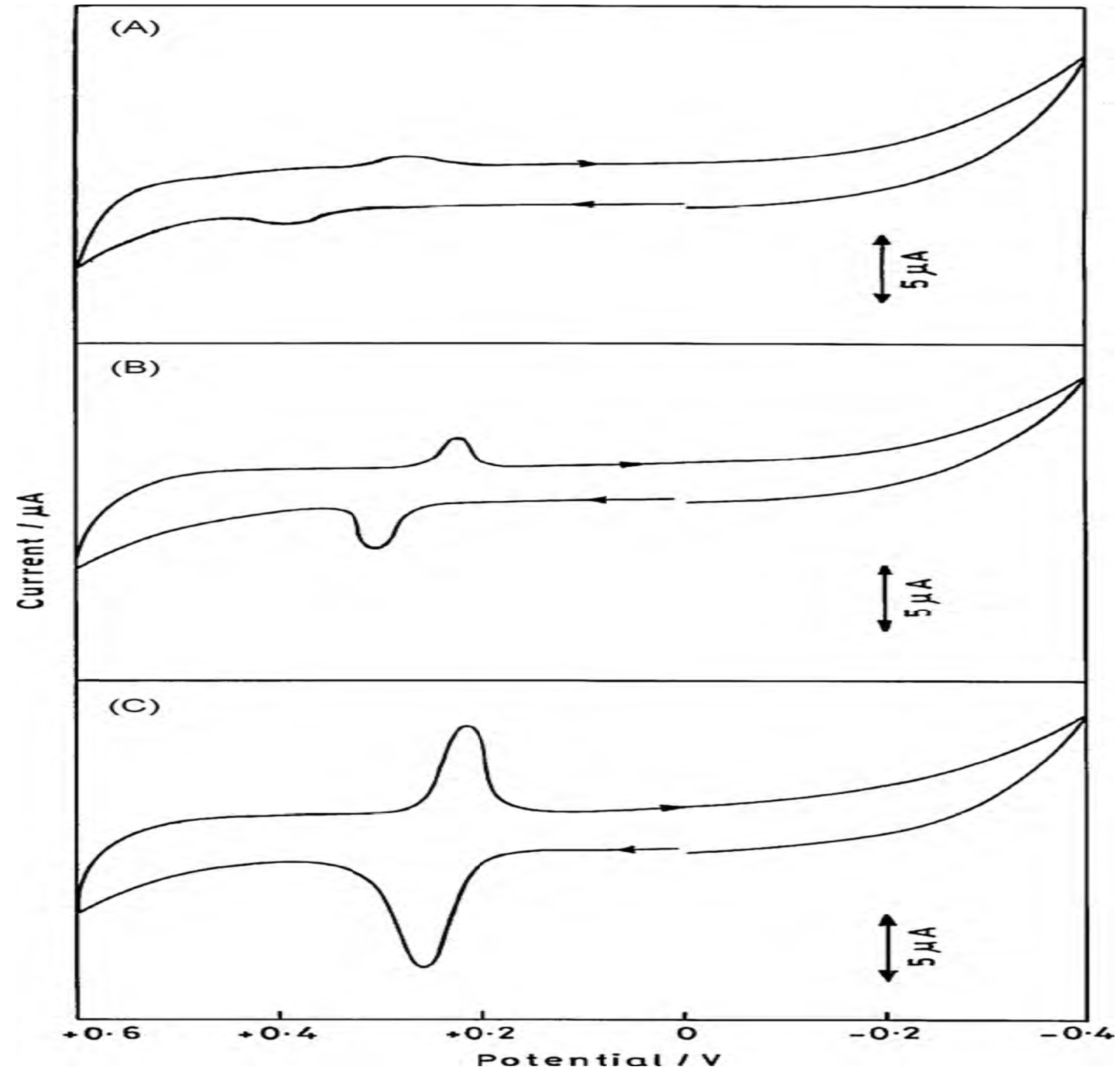

Fig. 1. Cyclic voltammograms obtained for 400nMparacetamol at pH 7.2 at (A) bare EPPGE, (B) MWNT modified EPPGE and (C) SWNT modified EPPGE at 50m Vs-1. 


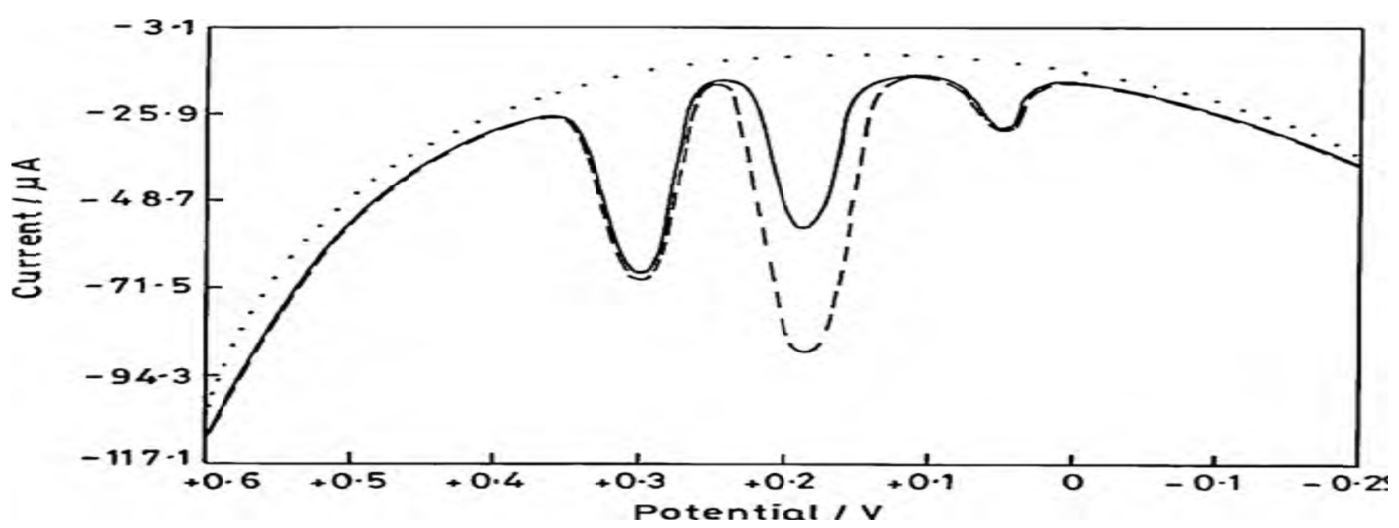

Fig. 2. A comparison of voltammograms observed for urine sample of patient being treated with paracetamol (-) and the patient sample spiked with paracetamol (-- ) at pH 7.2 at SWNT modified EPPGE. Background is represented as (...).

Table 1. Concentration of paracetamol in human urine after $4 \mathrm{~h}$ of paracetamol administration at SWNT modified EPPGE at $\mathrm{pH}$ 7.2

\begin{tabular}{|c|c|c|c|}
\hline & $\begin{array}{l}\text { Spiked } \\
(\mu \mathrm{M})\end{array}$ & $\begin{array}{l}\text { Detected } \\
(\mu \mathrm{M})^{\mathrm{a}}\end{array}$ & $\begin{array}{l}\text { Recovery } \\
(\%)\end{array}$ \\
\hline \multirow{4}{*}{$\begin{array}{l}\text { Sample } \\
1\end{array}$} & 0.00 & 4.23 & - \\
\hline & 1.00 & 5.36 & 102.48 \\
\hline & 3.00 & 7.29 & 100.83 \\
\hline & 5.00 & 8.94 & 96.86 \\
\hline \multirow{4}{*}{$\begin{array}{l}\text { Sample } \\
2\end{array}$} & 0.00 & 4.24 & - \\
\hline & 1.00 & 5.28 & 100.76 \\
\hline & 3.00 & 7.19 & 99.31 \\
\hline & 5.00 & 9.37 & 101.41 \\
\hline \multirow{4}{*}{$\begin{array}{l}\text { Sample } \\
3\end{array}$} & 0.00 & 4.25 & - \\
\hline & 1.00 & 5.18 & 98.67 \\
\hline & 3.00 & 7.06 & 97.38 \\
\hline & 5.00 & 9.54 & 103.13 \\
\hline
\end{tabular}

${ }^{a}$ The R.S.D. value for determination was less than $3.2 \%$ for $n=3$.

[4] P.F. Li, L.H. Liu, P. Ma, C.L. Ding, W.H. Tong, J.Y. Yang and A.J. Lan. Jiefangjun Yaoxue Xuebao, $25,33-37(2009$.

[5] T. Madrakian, A. Afkhami and M. Mohammadnejad. Anal. Chim. Acta, 645, 2529(2009).

[6] A.A. Shirkhedkar, A.M. Shaikh and S.J. Surana. Eurasian J. Anal. Chem., 3 , 244-253(2008).

[7] T. Nemeth, P. Jankovics, J. Nemeth-Palotas and H. Koszegi-Szalai. J. Pharm. Biomed. Anal., 47, 746-749(2008)

[8] R.N. Goyal, V.K. Gupta, M. Oyama and N. Bachheti. Electrochem. Commun., 7, 803807(2005).

[9] R.N. Goyal, V.K. Gupta, A. Sangal and N. Bachheti. Electrochem. Commun., 8, 6570(2006).

[10] R.T. Kachoosangi, G.G. Wildgoose and R.G. Compton. Anal. Chim. Acta, 618, 54-60(2008).

[11] P. Fanjul-Bolado, P.J. Lamas-Ardisana, D. Hernandez-Santos and A. Costa-Garcia. Anal. Chim. Acta, 638, 133-138(2009).

[12] Streeter, G.G. Wildgoose, L. Shao, R.G. Compton, Sens. Actuators B: Chem. 133 462466(2008).

\section{Acknowledgements}

The authors are thankful to the Council of Scientific and Industrial Research,New Delhi

\section{References}

[1] F. Zhao and M. Miao. Zhongguo Yaoye, 18,37(2009),

[2] N.H.S. Ahmida, M.S. Abu-Naja and Y.S.A. Doghman. Asian J. Chem., 21, 22332240(2009).

[3] H.T.T. Nguyen and T.T.V. Tong. Tap Chi Duoc Hoc, 49, 44-48(2009). 